\title{
Co-activation of hedgehog and AKT pathways promote tumorigenesis in zebrafish
}

\author{
Bensheng Ju1,5, Jan Spitsbergen ${ }^{3}$, Christopher J Eden ${ }^{4}$, Michael R Taylor ${ }^{5}$ and \\ Wenbiao Chen $* 1,2$
}

\begin{abstract}
Address: ${ }^{1}$ Vollum Institute, Oregon Health and Science University, Portland, Oregon 97239, USA, ${ }^{2}$ Department of Molecular Physiology \& Biophysics, Vanderbilt University Medical Center, Nashville, Tennessee, 37232, USA, ${ }^{3}$ Center for Fish Disease Research, Oregon State University, Corvallis, Oregon 97331, USA, ${ }^{4}$ Department of Developmental Neurobiology, St Jude Children's Research Hospital, Memphis, Tennessee 38105, USA and ${ }^{5}$ Department of Chemical Biology \& Therapeutics, St Jude Children's Research Hospital, Memphis, Tennessee 38105, USA

Email: Bensheng Ju - Bensheng.Ju@stjude.org; Jan Spitsbergen - spitsbej@onid.orst.edu; Christopher J Eden - Christopher.Eden@stjude.org; Michael R Taylor - Michael.Taylor@stjude.org; Wenbiao Chen* - wenbiao.chen@vanderbilt.edu

* Corresponding author
\end{abstract}

Published: 25 June 2009

Molecular Cancer 2009, 8:40 doi:10.1 186/1476-4598-8-40
Received: II November 2008

Accepted: 25 June 2009

This article is available from: http://www.molecular-cancer.com/content/8/l/40

(C) 2009 Ju et al; licensee BioMed Central Ltd.

This is an Open Access article distributed under the terms of the Creative Commons Attribution License (http://creativecommons.org/licenses/by/2.0), which permits unrestricted use, distribution, and reproduction in any medium, provided the original work is properly cited.

\begin{abstract}
The zebrafish has become an important model for cancer research. Several cancer models have been established by transgenic expression of human or mouse oncogenes in zebrafish. Since it is amenable to efficient transgenesis, zebrafish have immense potential to be used for studying interaction of oncogenes and pathways at the organismal level. Using the Gal4VPI6-UAS binary transgenic expression approach, we established stable transgenic lines expressing an EGFP fusion protein of an activated zebrafish Smoothened (Smoal-EGFP). Expression of the zebrafish SmoalEGFP itself did not lead to tumor formation either in founder fish or subsequent generations, however, co-expressing a constitutively active human AKTI resulted in several tumor types, including spindle cell sarcoma, rhabdomyoma, ocular melanoma, astrocytoma, and myoxma. All tumor types showed GFP expression and increased Patched I levels, suggesting involvement of zebrafish Smoal in tumorigenesis. Immunofluorescence studies showed that tumors also expressed elevated levels of phosphorylated AKT, indicating activation of the PI3K-AKT pathway. These results suggest that co-activation of the hedgehog and AKT pathways promote tumorigenesis, and that the binary transgenic approach is a useful tool for studying interaction of oncogenes and oncogenic pathways in zebrafish.
\end{abstract}

\section{Findings}

The Hedgehog (Hh) pathway is involved in cell fate determination and embryonic patterning during early vertebrate development, and is also implicated in tumorigenesis [1]. Activation of the Hh pathway underlies the majority of sporadic human basal cell carcinoma (BCC) [2]. Expression of sonic Hedgehog, constitutively active Smoothened, and transcription factors Gli1 and Gli2 in keratinocytes results in BCC in transgenic frog, mice, and human skin [3-6]. Since zebrafish have emerged as a promising vertebrate system to model human cancers [7-9], we decided to determine whether activation of the Hh pathway in transgenic zebrafish could render them prone to developing BCC. We generated the zebrafish version of activated Smoothened using site-specific mutagenesis of wild type smoothened cDNA [SmoW514L, referred as Smoa1 hereafter, see Additional file 1]. To facilitate observation of the tumorigenesis proc- 
esses, we tagged the zebrafish Smoa1 with C-terminal EGFP. We expressed it under the control of a $C M V$ promoter, and observed GFP expression by 5 hours post fertilization (hpf). To assess the effect of Smoa1-EGFP expression on the Hh signalling pathway, we analyzed the expression of patched 1 (ptc1) [10], a marker for Hh activity, by in situ hybridization. We detected ectopic expression of ptc1 $(\mathrm{n}=30)$ in the $T g(C M V$ :smoa1-EGFP) injected, but not in non-injected control embryos (Fig. 1A, 1B respectively), indicating Smoa1-EGFP could still activate the Hh pathway.

We further generated a binary transgenic construct based on the Gal4VP16-UAS system [11] [see Additional file 2]. The zebrafish cytokeratin 4 (krt4) promoter was used to drive expression of Gal4VP16 as it has been shown to direct EGFP expression exclusively in epithelial cells [12]. Injection of the Tg(krt4:Gal4VP16;14 × UAS:smoa1-EGFP) construct into 1-cell stage embryos led to observable levels of EGFP expression starting at about 5 hpf. We analyzed more than 100 founder fish over a period of one and a half years, but did not find any tumor. Adult founder fish were crossed to wild-type fish, and two male founder fish were found to carry the transgene in their germline. These lines were designated as line A and line $\mathrm{B}$. Both lines did not show observable EGFP expression, but in situ hybridization using a GFP antisense probe detected low levels of GFP expression in skin epithelial cells [see Additional file 3]. Both lines were also crossed with a $\operatorname{Tg}(14 \times$ UAS:tdTomato) reporter line that we generated in the rose background (unpublished results) to verify their expression patterns [13]. Line A showed epithelial restricted expression [see Additional file 3], while line B showed patchy epithelial expression and ectopic expression in neuronal cells of the brain, spinal cord, and trunk muscles

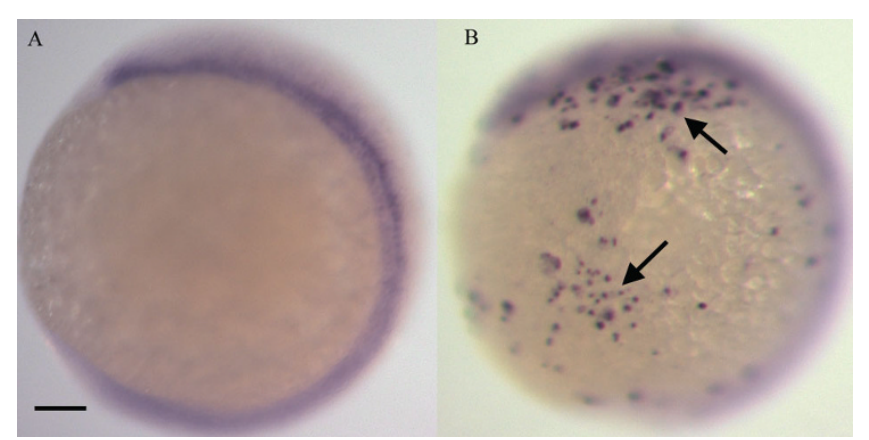

\section{Figure I}

Expression of zebrafish Smoal activated the $\mathbf{H h}$ pathway. A, whole-mount in situ hybridization of a control I 2 hpf embryo showing ptcl expression in adaxial structures. $\mathrm{B}$, a I2 hpf transgenic embryo expressing CMV-smoa I-EGFP showed ectopic expression of ptcl (Arrows). Scale bars, 100 $\mu \mathrm{m}$.
(Data not shown). Fish positive for tdTomato expression in both lines were raised to adulthood and no tumor were found even when the fish reached one and a half years old. To further characterize the expression pattern for line A, an adult fish at two months old was sacrificed and paraffin sections were used for immunofluorescence against GFP. Expression was found predominantly in the skin epithelia [see Additional file 3], which was expected since the promoter driving the transgenic expression was a skinspecific krt4 promoter. Expression of GFP was also found in the retina ganglion cell layer [see Additional file 3]. We did not find prominent expression of GFP in other tissues or organs.

Since the Tg(krt4:Gal4V16;14 × UAS-smoa1:EGFP) fish did not develop tumors at one and a half years of age, we decided to test whether other oncogenes could collaborate to promote tumorigenesis. We chose to use transgenic line A as it showed rather specific expression in epithelia in comparison to line B. A recent report has shown that PI3K and AKT are essential for SHH signalling [14]. As a first test of our co-expression strategy, we expressed the wildtype and the constitutively active human $A K T 1$ by injecting $\mathrm{Tg}(U A S: h A K T 1)$ or $\mathrm{Tg}(U A S: m y r h A K T 1)$ plasmid DNA into progeny of F1 siblings of the Tg(krt4:Gal4V16;14 $\times$ UAS-smoa1-EGFP) line A fish. Both the smoa1 and AKT1 genes were under control of the $14 \times U A S$ promoter, so their expression was driven by the same krt4 promoter through Gal4VP16. While no tumor was found in the resultant F2 fish injected with $T g(U A S: h A K T 1)$, various tumor types in the trunk, the eye, and the head region were observed in the F2 fish injected with Tg(UAS:myrhAKT1). The tumor types identified include a case of spindle cell sarcoma, rhabdomyoma, ocular melanoma, myoxma, and several cases of astrocytoma and glioblastoma (Fig. 2 and Table 1). These tumor types were diagnosed according to histological criteria used in mammals [15]. GFP expression was found exclusively in the tumors, due to either the additive effect of a large number of weakly EGFP-positive cells or the loss of mechanisms that suppress Smoa1-EGFP levels, or both. In any case, the detection of EGFP expression in tumors indicated the involvement of the zebrafish Smoa1 in tumorigenesis (Fig. 2B, 2E). Overall, nearly $10 \%$ of injected fish developed tumors before reaching 3 months of age. Surprisingly, the rest of the fish were tumor-free at 18 months of age, at which we stopped the experiment. We also identified fish that carried both zebrafish smoa1 and the oncogenic human AKT1 in their germline. We crossed two of these fish to each other, and found 3 out of 15 of the offspring developed tumors at around 2 months of age, including one case of astrocytoma in the lower-trunk region (Fig. $2 \mathrm{H}$ ), the other two cases were not diagnosed. In contrast, expression of either wild-type or constitutively active human AKT1 alone, driven by the same krt4 pro- 


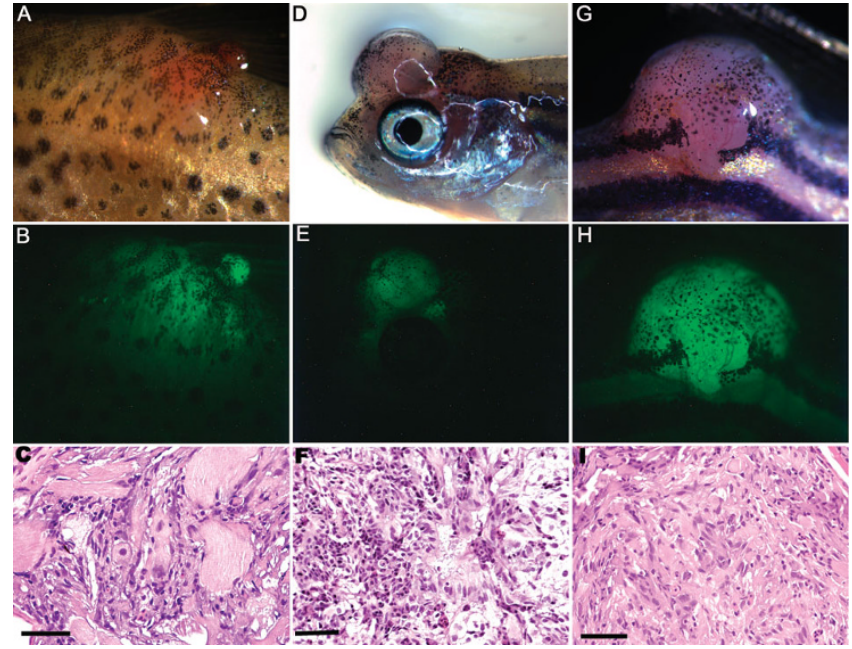

Figure 2

Zebrafish tumors induced by co-expression of zebrafish Smoal and constitutively active human AKTI. A, B and C, a I2-week-old fish with rhabdomyoma (A), showing GFP expression exclusively in the tumor (B); D, $\mathrm{E}$ and $\mathrm{F}$, a 6-week-old fish with glioblastoma; G, H and I, a 8week-old double transgenic fish with astrocytoma in the lower trunk region. Scale bars, $50 \mu \mathrm{m}$.

moter, did not induce tumors. In fact, even when we expressed the constitutively active human AKT1 driven by a strong ubiquitous -actin promoter, no tumor was found in more than 50 fish over an 18 months period. Thus, the tumors likely resulted from collaboration between the constitutively active hAKT1 and Smoa1.

Activation of the PI3K-AKT pathway leads to phosphorylation of AKT1 [14]. To determine whether phospho-
AKT1 levels were increased in the tumors, we performed immunofluorescence studies on a 3-week-old fish with a trunk tumor showing strong GFP expression and on a 12week-old fish with an ocular tumor showing weak GFP expression. Immunofluorescence analysis indicated that both tumors had significantly higher levels of phosphorylated AKT (Fig. 3B, 3D) when compared to age-matched, tumor-free transgenic fish (Fig. 3A, 3C). To demonstrate that both the SHH and AKT pathways were activated in a same tumor, paraffin sections of the astrocytoma as shown in Fig. 2G was used for immunofluorescence study against Patched 1 and phosphorylated AKT. As shown in Fig. 3F and 3G, the tumor had elevated levels of both Patched 1 and phosphorylated AKT, indicating that both pathways were activated in this tumor. These results suggest that the tumors resulted from collaborative expression of both the zebrafish Smoa1 and the constitutively active human AKT1.

Here we demonstrated that several tumor types in zebrafish can be induced by transgenic expression of oncogenes, most likely due to co-expression of an oncogenic zebrafish Smoa1 and the constitutively active human AKT1. It was surprising that we did not find any skin tumor, as activation of either the Hh or AKT pathway $[16,17]$ leads to skin hyperplasia and skin tumors in mouse. We also generated a transgenic line using the TetOff system that expressed the Smoa1-EGFP driven by a 5 $\mathrm{kb}$ zebrafish cytokeratin 5 (krt5) gene promoter. This transgenic line expressed higher levels of zebrafish Smoa1, but we did not find any skin tumor in fish that reached two years of age (Data not shown). It is possible that either: 1) the level of Smoa1 expression was below the threshold for tumorigenesis; 2) the Smoa1-EGFP fusion protein was not potent enough; 3) the krt4 and krt5 promoters were not

Table I: Tumors induced by co-expression of zebrafish smoal and constitutively active human AKT I

\begin{tabular}{|c|c|c|c|c|}
\hline Tumor location (cases) & Fish age (weeks) & GFP expression & Elevated phospho-AKTI & Diagnoses \\
\hline \multirow[t]{4}{*}{ Trunk (4) } & 3 & Yes & ND & Rhabdomyoma \\
\hline & 4 & Yes & Yes & ND \\
\hline & 6 & Yes & ND & ND \\
\hline & 12 & Yes & ND & Rhabdomyoma \\
\hline \multirow[t]{3}{*}{ Eye (3) } & 4 & Yes & ND & Ocular Melanoma \\
\hline & 12 & Yes & Yes & Astrocytoma \\
\hline & 12 & Yes & ND & Astrocytoma \\
\hline \multirow[t]{4}{*}{ Head (4) } & 6 & Yes & ND & Glioblastoma \\
\hline & 6 & Yes & ND & Glioblastoma \\
\hline & 8 & Yes & ND & ND \\
\hline & 12 & Yes & ND & Astrocytoma \\
\hline \multirow[t]{2}{*}{ Others (2) } & 4 & Yes & ND & Myxoma \\
\hline & 8 & Yes & ND & Spindle cell sarcoma \\
\hline
\end{tabular}

*The tumors were found from 4 batches of injection involving 147 injected F2 fish that had survived beyond 2-week-old. Fish were observed for one and a half years, no other tumors were found in fish more than 3 months of age. ND: Not determined. 

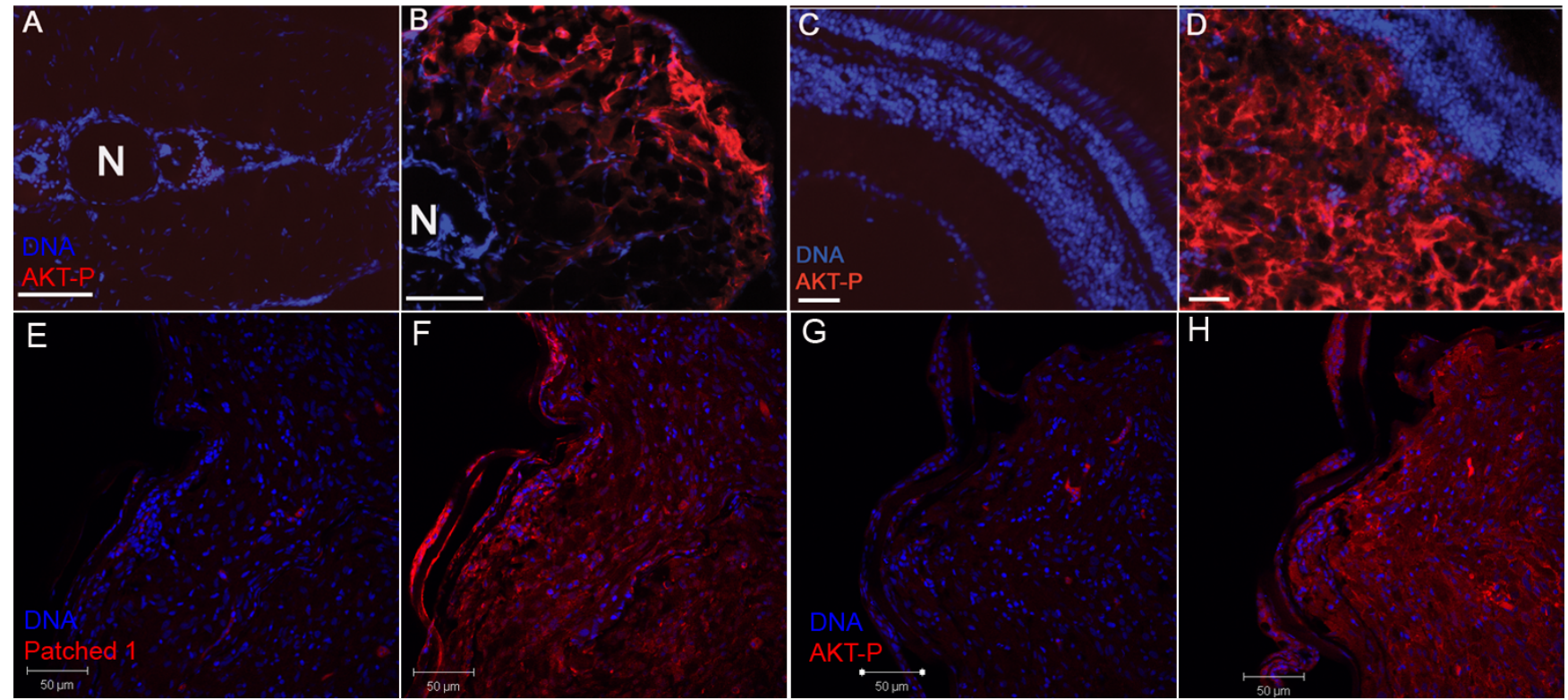

Figure 3

Elevated phospho-AKTI and Patched I levels in zebrafish tumors. A, B, a 3-week-old transgenic fish with trunk tumor (B) and its age-matched tumor-free fish (A). C, D, a I2-week-old fish with an eye tumor (D) and its age-matched tumor free fish (C). Immunofluorescence was done on cryosections for the above fish. E-H, an astrocytoma from a double transgenic fish showed elevated levels of both Patched I (F) and phosphorylated AKT (H). Immunofluoresence was done on paraffin sections for this tumor. Negative controls for Patched I (E) and phosphorylated AKT $(G)$ were treated the same way except no primary antibodies were added. N, Notochord; AKT-P, phosphorylated AKT. Scale bars, I00 $\mu \mathrm{m}$ for A-D, $50 \mu \mathrm{m}$ for E-H.

active in keratinocyte progenitors; or 4) the combinations thereof. Alternatively, zebrafish skin may be quite refractory to developing basal cell carcinoma in comparison to frog and mammals, due to differences either in structure or in tumorigenic mechanisms. As for the other tumor types, especially the neuronal tumors, aberrant activation of the Hh pathway has been implicated in medulloblastoma and glioma [18]. Studies on AKT pathway implicate it not only in skin tumors, but also in glioblastoma [19]. Furthermore, the Akt signalling pathway contributes to SHH-induced medulloblastoma formation [20]. Therefore, it is conceivable that tumor types of neuronal origin may be induced in fish. But how exactly these tumors were induced in our transgenic fish is unknown. Immunofluorescence study showed that transgenic fish expressed the fusion protein not only in skin epithelia, but also in retina, which was not found in a previous study using the same promoter. An enhancer-trapping event might have occurred that led to ectopic expression of low levels of zebrafish Smoa1 in neuronal progenitor cells, which made the cells prone to developing tumors. Expression of a second oncogene, the constitutively active human AKT1, in the same cell types eventually led to tumorigenesis. It is also possible that integration of the transgenic construct into the zebrafish genome somehow activated an endogenous oncogene or inactivated a certain tumor suppressor gene, therefore contributed to tumor formation. We tried to determine the insertion copy numbers and the insertion sites in Line A fish through linker-mediated PCR and inverse PCR. We only detected one insertion, which was integrated at an intergenic region on zebrafish chromosome 11 (Data not shown). We do not know the effects of this integration on tumorigenesis in this particular transgenic line.

Cancers result from progressive accumulation of mutations in multiple cancer genes [21,22]. How different cancer genes interact with each other leading to different types of cancer is still a challenging subject. So far, oncogenic interactions have largely been studied in cultured cells or in mouse models. As a complement to these studies, the zebrafish provides a powerful model system to study interactions between different cancer genes and pathways at the organismal level because it is amenable to highly-efficient and cost-effective transgenic strategies $[23,24]$. Binary transgenic technology, such as the Gal4VP16-UAS system, the Tet-On and Tet-Off system [25], and the mifepristone inducible LexPR system [26] work efficiently in zebrafish, making it possible to express multiple oncogenes in the same tissue or cell type under the control of the same non-endogenous transcription factor. A limitation for using these binary transgenic approaches, as we have learned from our experience, is that stable lines carrying oncogenes may have diminished 
survivability. Therefore, their potential to be used for collaborative studies with other oncogenes may be somewhat compromised. Another challenge will be to untangle the role of individual oncogenes in the tumor formation and progression processes.

In conclusion, we provide in vivo evidence that co-expression of the zebrafish Smoa1 and the constitutively active human AKT1 lead to tumorigenesis in zebrafish, establishing that the binary transgenic approach is a useful tool for studying collaboration between oncogenes and oncogenic pathways in the zebrafish model.

\section{Materials and methods Zebrafish and maintenance}

Zebrafish (Danio rerio) were maintained in Aquatic Habitats systems (Apopka, FL) on a 14 to 10 light dark cycle using a protocol approved by OHSU IACUC. The leopard long fin (TL), and the rose mutant fish which has a mutation in the endothelial receptor $b 1$ gene causing light pigmentation were used. Embryos were reared in $0.3 \times$ Danieau's solution at $28.5^{\circ} \mathrm{C}$

\section{DNA constructs and microinjection}

The transgenic DNA constructs are based on either the miniTol2 vector (denoted T2) or the I-Sce I meganuclease vector (denoted I) [see additional file 1]. The Tg(krt4:Gal4VP16;14 × UAS:smoa1-EGFP) transgenic construct contains a $2.2 \mathrm{~kb}$ zebrafish krt4 promoter, the Gal4VP16-UAS sequence from pEF-GVP-UG, and coding sequence for an activated zebrafish smo fused to EGFP at the C-terminus. The IU-mCherry plasmid, which contains $14 \times$ UAS and E1b sequence from pUG and mCherry coding sequence, is the basic vector from which other UASdriven vectors are derived, including pIU-hAKT1 and pIUmyrhAKT1 (myristylated human AKT1 lacking the Pleckstrin Homology Domain sequence, referred to as constitutively active human AKT1). To deliver transgenes for transient expression or to establish stable lines using Tol2based plasmids, about $1 \mathrm{nl}$ sterile isotonic saline solution containing transgene DNA (30 $\mathrm{ng} / \mu \mathrm{l})$ and transposase RNA (30 $\mathrm{ng} / \mu \mathrm{l})$ was injected into fertilized eggs of the leopard strain. For the meganuclease-based constructs, the vector was mixed with $I-S c e I$ meganuclease and $30 \mathrm{pg}$ of plasmid in $1 \mathrm{nl}$ of the mixture was injected. Transgenic lines were identified by PCR using a zebrafish smoothened forward primer (5'-GGAAAGGAACAAACTTTGGATG-3') and an EGFP reverse primer (5'-CTGAACTTGTGGCCGTTTACGTC-3').

\section{Histological study of zebrafish tumors}

Zebrafish with tumors were fixed in $4 \%$ paraformaldehyde at $4{ }^{\circ} \mathrm{C}$ for $24-48$ hours, washed with PBST and decalcified in $0.5 \mathrm{M}$ EDTA for up to 7 days depending on size. Tissues were dehydrated in a series of graded ethanol solutions and xylene, then embedded in paraffin. Orientation of the fish depended on location of the lesions. For fish with no gross lesions, individuals were cut in half sagittally just to the left of midline and both halves of the fish were placed into the cassette for sectioning. For small fish, serial sections were prepared. Sections were 4-6 $\mu \mathrm{m}$ thick and were stained with hematoxylin and eosin. Since standardized diagnostic criteria for fish tumors is not yet available, diagnostic criteria for other species, such as human and mouse were used to identify zebrafish tumors.

\section{Immunofluorescence study of zebrafish tumors}

For cryosections, zebrafish with tumors were fixed in $4 \%$ paraformaldehyde at $4{ }^{\circ} \mathrm{C}$ for 24 hours, washed with PBST. Tumor tissues were first immersed in $15 \%$ glucose for 1 hour, followed by immersion in 30\% sucrose overnight. They were then embedded in O.C.T compound and sectioned at $12 \mu \mathrm{m}$ thickness. For paraffin sections, fish were treated as mentioned in histological study of zebrafish tumors. A rabbit anti-GFP polyclonal antibody (Invitrogen, 1:500), a rabbit anti-phospho-AKT (Thr308) antibody (C31E5, Cell Signalling Technology, 1:200), and a goat anti-Patched 1 (zebrafish) antibody (Everest Biotech, 1:200) were used for immunofluorescence studies of the tumors. Images were acquired with an inverted Zeiss microscope equipped with a CCD camera and AxioVision software. All images are processed in Photoshop.

\section{Abbreviations}

PI3K: Phosphoinositide 3-kinases; AKT1: v-akt murine thymoma viral oncogene homolog 1; UAS: upstream activation sequence; CMV: Cytomegalovirus.

\section{Competing interests}

The authors declare that they have no competing interests.

\section{Authors' contributions}

$\mathrm{BJ}$ and $\mathrm{WC}$ conceived, planned the experiments. BJ made the transgenic constructs and generated transgenic fish. JS identified the tumors. CJE and MRT did immunofluorescence studies on tumors. All authors read and approved the manuscripts.

\section{Additional material}

\section{Additional file 1}

Amino acid sequence alignment of mouse and zebrafish Smoothened. The alignment indicated that the W514 of zebrafish Smoothened is the W539 equivalent of mouse Smoothened. It was mutated to $L$ in the active forms (highlighted in red). Accession numbers for zebrafish and mouse Smoothened are [NP_571102] and [NP_795970], respectively. Click here for file

[http://www.biomedcentral.com/content/supplementary/14764598-8-40-S1.doc] 


\begin{abstract}
Additional file 2
Overall strategy for co-expression of oncogenes in zebrafish. Stable transgenic lines expressing zebrafish Smoa1 were generated using a Tol2based vector (A). Constitutively active human AKT1 (myrhAKT1) was incorporated into a meganuclease-based vector (B). The zebrafish $\mathrm{krt} 4$ promoter could simultaneously activate smoa1-EGFP and myrhAKT1 expression through Gal4VP16-UAS.

Click here for file

[http://www.biomedcentral.com/content/supplementary/14764598-8-40-S2.tiff]

\section{Additional file 3}

Expression patterns of transgenic line $A$. The data indicated that the cytokeratin 4 promoter drove epithelial cells-specific expression (arrows) of smoa1-EGFP as shown by in situ hybridization against EGFP in $12 \mathrm{hpf}$ F1 embryos (A), and of tdTomato in a $24 \mathrm{hpf}$ embryo generated by crossing the $\mathrm{Tg}(\mathrm{krt} 4: \mathrm{Gal} 4 \mathrm{VP} 16 ; 14 \times$ UAS:smoa1-EGFP) and

$\mathrm{Tg}$ (UAS:tdTomato) transgenic fish (B). At adult stage, GFP was detected predominantly in skin epithelial cells $(C$, arrow) and the retinal ganglion cells ( $D$, arrow).

Click here for file

[http://www.biomedcentral.com/content/supplementary/14764598-8-40-S3.tiff]
\end{abstract}

\section{Acknowledgements}

We would like to thank Phillip Stork from Vollum Institute and Peter Rotwein from the Department of Biochemistry, Oregon Health and Science University for providing the oncogenes, Koichi Kawakami (National Institute of Genetics, Shizuoka, Japan) for the miniTol2 vector and Jochen Wittbrodt (Developmental Biology Programme, European Molecular Biology Laboratory, Heidelberg, Germany) for the meganuclease vector. The authors also thank Pinxuan Yu for fish care, Samuel Connell and Jennifer L. Peters from Imaging Center of St Jude Children's Hospital for assistance with image capturing and processing. This research is supported by NIH grant EYOI 6092 to Wenbiao Chen and by U.S. Public Health Service Grant ESO I 3 I 24 from the National Institutes of Environmental Health to Jan Spitsbergen.

\section{References}

I. Jacob L, Lum L: Deconstructing the hedgehog pathway in development and disease. Science 2007, 31 8:66-68.

2. Boukamp P: Non-melanoma skin cancer: what drives tumor development and progression? Carcinogenesis 2005, 26:1657-1667.

3. Dahmane N, Lee J, Robins P, Heller P, Ruiz i, Altaba A: Activation of the transcription factor Glil and the Sonic hedgehog signalling pathway in skin tumours. Nature 1997, 389:876-88।.

4. Fan H, Oro AE, Scott MP, Khavari PA: Induction of basal cell carcinoma features in transgenic human skin expressing Sonic Hedgehog. Nat Med 1997, 3:788-792.

5. Grachtchouk M, Mo R, Yu S, Zhang X, Sasaki H, Hui CC, Dlugosz AA: Basal cell carcinomas in mice overexpressing Gli2 in skin. Nat Genet 2000, 24:216-2I7.

6. Xie J, Murone M, Luoh SM, Ryan A, Gu Q, Zhang C, Bonifas JM, Lam CW, Hynes M, Goddard A, et al.: Activating Smoothened mutations in sporadic basal-cell carcinoma. Nature 1998, 39 I:90-92.

7. Beckman M: Zebrafish take the stage in cancer research. J Natl Cancer Inst 2007, 99:500-50I.

8. Berghmans S, Jette C, Langenau D, Hsu K, Stewart R, Look T, Kanki JP: Making waves in cancer research: new models in the zebrafish. Biotechniques 2005, 39:227-237.

9. Goessling W, North TE, Zon LI: New waves of discovery: modeling cancer in zebrafish. J Clin Oncol 2007, 25:2473-2479.
10. Concordet JP, Lewis KE, Moore JW, Goodrich LV, Johnson RL, Scott MP, Ingham PW: Spatial regulation of a zebrafish patched homologue reflects the roles of sonic hedgehog and protein kinase $\mathbf{A}$ in neural tube and somite patterning. Development 1996, I 22:2835-2846.

II. Davison JM, Akitake CM, Goll MG, Rhee JM, Gosse N, Baier H, Halpern ME, Leach SD, Parsons MJ: Transactivation from Gal4-VPI6 transgenic insertions for tissue-specific cell labeling and ablation in zebrafish. Dev Biol 2007, 304:8I I-824.

12. Gong Z, Ju B, Wang X, He J, Wan H, Sudha PM, Yan T: Green fluorescent protein expression in germ-line transmitted transgenic zebrafish under a stratified epithelial promoter from keratin8. Dev Dyn 2002, 223:204-215.

13. Parichy DM, Mellgren EM, Rawls JF, Lopes SS, Kelsh RN, Johnson SL: Mutational analysis of endothelin receptor bl (rose) during neural crest and pigment pattern development in the zebrafish Danio rerio. Dev Biol 2000, 227:294-306

14. Riobo NA, Lu K, Ai X, Haines GM, Emerson CP Jr: Phosphoinositide 3-kinase and Akt are essential for Sonic Hedgehog signaling. Proc Natl Acad Sci USA 2006, 103:4505-45I0.

15. Koestner A, Bilzer T, Fatzer R, Schulman FY, Summers BA, Van Winkle TJ: Histological Classification of Tumors of the Nervous System of Domestic Animals. World Health Organization/Armed Forces Institute of Pathology/American Registry of Pathology 1999.

16. Segrelles C, Lu J, Hammann B, Santos M, Moral M, Cascallana JL, Lara MF, Rho O, Carbajal S, Traag J, et al.: Deregulated activity of Akt in epithelial basal cells induces spontaneous tumors and heightened sensitivity to skin carcinogenesis. Cancer Res 2007, 67: $10879-10888$

17. Lu J, Rho O, Wilker E, Beltran L, Digiovanni J: Activation of epidermal akt by diverse mouse skin tumor promoters. Mol Cancer Res 2007, 5: 1342-1352.

18. Wicking C, Smyth I, Bale A: The hedgehog signalling pathway in tumorigenesis and development. Oncogene 1999, 18:7844-785।.

19. Knobbe CB, Reifenberger G: Genetic alterations and aberrant expression of genes related to the phosphatidyl-inositol-3'kinase/protein kinase B (Akt) signal transduction pathway in glioblastomas. Brain Pathol 2003, I 3:507-518.

20. Rao G, Pedone CA, Del Valle L, Reiss K, Holland EC, Fults DW: Sonic hedgehog and insulin-like growth factor signaling synergize to induce medulloblastoma formation from nestinexpressing neural progenitors in mice. Oncogene 2004, 23:6156-6162.

21. Hanahan D, Weinberg RA: The hallmarks of cancer. Cell 2000, 100:57-70.

22. Vogelstein B, Kinzler KW: Cancer genes and the pathways they control. Nat Med 2004, 10:789-799.

23. Kawakami K: Tol2: a versatile gene transfer vector in vertebrates. Genome Biol 2007, 8(SuppI I):S7.

24. Grabher C, Joly JS, Wittbrodt J: Highly efficient zebrafish transgenesis mediated by the meganuclease I-Scel. Methods Cell Biol 2004, 77:38I-40I.

25. Huang CJ, Jou TS, Ho YL, Lee WH, Jeng YT, Hsieh FJ, Tsai HJ: Conditional expression of a myocardium-specific transgene in zebrafish transgenic lines. Dev Dyn 2005, 233:1294-1303.

26. Emelyanov A, Parinov S: Mifepristone-inducible LexPR system to drive and control gene expression in transgenic zebrafish. Dev Biol 2008, 320: I|3-121.

Publish with Bio Med Central and every scientist can read your work free of charge

"BioMed Central will be the most significant development for disseminating the results of biomedical research in our lifetime. "

Sir Paul Nurse, Cancer Research UK

Your research papers will be:

- available free of charge to the entire biomedical community

- peer reviewed and published immediately upon acceptance

- cited in PubMed and archived on PubMed Central

- yours - you keep the copyright 